\title{
THORACIC EPIDURAL ANESTHESIA FOR MODIFIED RADICAL MASTECTOMY-IN TYPE2 DIABETES MELLITUS PATIENT
}

Vishwanath R. Hiremath ${ }^{1}$

\section{HOW TO CITE THIS ARTICLE:}

Vishwanath R. Hiremath. "Thoracic Epidural Anesthesia for Modified Radical Mastectomy-In Type 2 Diabetes Mellitus Patient". Journal of Evolution of Medical and Dental Sciences 2014; Vol. 3, Issue 70, December 15;

Page: 15002-15006, DOI: $10.14260 /$ jemds/2014/4017

ABSTRACT: Modified radical mastectomy (MRM), the standard oncologic surgical procedure of the carcinoma of breast is routinely performed under general anesthesia. Carcinoma breast patients are considered to be at high risk for anesthesia due to high possibility of perioperative complications and mortality when associated with long standing type 2 Diabetes Mellitus (DM) with other co morbidities. Cardiac complications due to macro vascular and micro vascular involvement can pose a real threat. Here we present a case report of successful perioperative management of modified radical mastectomy only with thoracic epidural anesthesia (TEA) in a case of carcinoma breast with long standing type $2 \mathrm{DM}$ and hypertension. A 71year old female, a known case of DM since 7years with co morbidity; hypertension having a carcinoma breast was scheduled for modified radical mastectomy. Continuous epidural anesthesia was administered at T4-5 level. Local anesthetic supplementation titrated as per the demands of surgery and good postoperative analgesia maintained for 48 hours. Long standing DM with hypertension can lead to perioperative morbidity and mortality due to cardiac complications. However, Thoracic epidural anesthesia (TEA) reduces cardiac and sympathetic activity and thereby improves perioperative function of vital organs and reduces postoperative morbidity and mortality. The procedure can lead to prompt recovery with additional benefit of prolonged post-operative analgesia. TEA proved to be an excellent anesthetic technique for MRM in patient with long standing DM.

KEYWORDS: Modified radical mastectomy, Carcinoma breast, Thoracic epidural anesthesia, Diabetes mellitus.

INTRODUCTION: The incidence of breast malignancy is increasing worldwide at an alarming rate and is a leading cause of mortality in women. In India, prevalence of carcinoma breast varies from 1231 cases per 100, 000 women.(1) Modified radical mastectomy (MRM), is the standard surgical procedure of choice in these patients. MRM is usually performed under general anesthesia; almost always combining intravenous and inhalational agents. The drawback of general anesthesia includes inadequate pain control due to lack of residual analgesia, high incidence of nausea and vomiting, stress of anesthesia and surgery, and increasing the length of hospital stay.(2) Further, general anesthesia in cancer patients may depress the immune system.(3) Patients of long standing type 2 DM with other co morbidities are at high risk of perioperative morbidity due to cardiovascular complications. Thoracic epidural anesthesia is frequently used in cosmetic surgeries of breast (4) and there are also reports on its use in oncologic surgeries of the breasts. ${ }^{(5-7)}$ However, these procedures are not commonly practiced. In the present report we describe successful anesthetic management of a case of long standing type 2 DM with hypertension under TEA in designated case of carcinoma of breast for modified radical mastectomy. 


\section{CASE REPORT}

CASE REPORT: A 71years old female patient weighing $72 \mathrm{~kg}$ presented to surgical out- patient department (OPD) with chief complaint of lump in the right breast since 2 years gradually increasing in size. On clinical examination, a hard lump of $5 \times 5 \mathrm{~cm}$, with hard irregular surface, not fixed to the skin, with no history of any discharge from nipple, and enlarged axillary lymph nodes on the right side. Fine needle aspiration cytology revealed presence of malignant cells and patient was scheduled for MRM.

Pre-operatively, apart from exertional dyspnoea (Grade-II), she was asymptomatic. Physical examination revealed moderate obesity, pulse rate of $80 \mathrm{~min}^{-1}$, blood pressure 120/78 $\mathrm{mm} \mathrm{Hg}$ with room air oxygen saturation (Sp02)95\%. With no rise in jugular venous pressure (JVP). Her lungs were clear to auscultation and cardiac examination showed regular rate and rhythm. Chest radiograph showed increased normal lung fields. Electrocardiography revealed left ventricular hypertrophy (LVH). Two- dimensional Echocardiography (2D-Echo) with color Doppler revealed mild LVH with impaired left ventricular relaxation and low LV systolic function. Her hemoglobin was $12 \mathrm{~g} \%$, Her fasting blood glucose was $163 \mathrm{mg} \%$, PPBS $201 \mathrm{mg} \%$ and HbA1c $7.5 \%$ suggesting poor blood glucose control. Her lipid control, serum electrolytes, serum creatinine and blood urea were within normal limits. Considering long standing DM, hypertension and cardiac impairment patient was accepted as Grade III ASA (American Society of Anesthesiologists). Meanwhile patient blood glucose was optimized with injection Actrapid insulin for 3 days. Tablet Metformin discontinued 24 hours prior to the operation. Morning dose of insulin omitted and 5\% dextrose with $10 \mathrm{~m}$ mol of potassium chloride and 5 Units of Insulin (GIK) started at $100 \mathrm{ml}$ per hour at $6 \mathrm{AM}$ in the morning. Anti-hypertensive medication supplemented with sips of water at 6AM in the morning.

The patient was taken to the operation theatre with American Society Anesthesiologists (ASA) Grade III physical status with informed consent, explaining the epidural anesthetic technique. In the operation theatre, a good intravenous (IV) access was secured with a wide bore cannula and normal saline (NS) infusion started for maintenance and replacement of fluids. Standard monitors; NIBP, Pulse oximeter probe and ECG leads connected. The surgery planned under the thoracic epidural anesthetic technique. Blood sugar recording at the beginning of surgery was $140 \mathrm{mg} / \mathrm{dl}$.GIK started at $100 \mathrm{ml} /$ hour.

Anesthetic Technique: In the sitting position, with all aseptic precautions land marks identified and T4-5 level demarcated. Local anesthetic agent 2\% plain lignocaine was infiltrated with $26 \mathrm{G}$ needle at the site. Subsequently, Tuohy 18G B Braun needle was introduced and epidural space identified by loss of resistance (LOR) technique. Epidural catheter introduced $4 \mathrm{~cm}$ into the epidural space through the Tuohy needle in the cephaloid direction. Catheter was fixed and $3 \mathrm{ml}$ test dose of lignocaine $2 \%$ with adrenaline 1:200000 given. Patient was observed for 3 minutes for intravenous or intrathecal placement. Confirming the proper placement of the catheter initial dose of $8 \mathrm{ml}$ of $0.75 \%$ Ropivacaine with 50ug of Fentanyl injected through the catheter which resulted in bilateral anesthesia of thoracic wall; from the lower border of clavicle to the inferior costal margin in 20 minutes. Following the satisfactory anesthesia and analgesia surgery was initiated. Surgeons were happy with the surgical field and blood loss was minimal. At the time of axillary exploration, midazolam $1 \mathrm{mg}$ and fentanyl 50ug supplemented intravenously. Surgeon infiltrated the area with $5 \mathrm{ml}$ of $0.75 \%$ ropivacaine. Surgery lasted for $2 \mathrm{hr}$ and 15 minutes without any significant changes in heart rate and blood pressure. Post operatively, patient was observed in the post-operative recovery room for $1 \mathrm{hr}$ and then shifted to the surgical intensive care unit ( SICU). Pain relief achieved with $0.2 \%$ Ropivacaine 


\section{CASE REPORT}

with Fentanyl 25ug, total of $8 \mathrm{ml}$ supplemented through the epidural catheter 12 hourly for 48 hours. Remaining part of postoperative stay was uneventful. She remained clinically stable and was discharged on the $6^{\text {th }}$ postoperative day.

DISCUSSION: People with diabetes undergoing surgery have an almost 50\% greater chance of postoperative mortality and morbidity than those with normal glucose tolerance. Mortality rates in diabetics following surgery are estimated to be 5 times greater than in non-diabetic counter parts; often due to end organ damage and infections.Thoracic epidural anesthesia (TEA) has been established as a cornerstone in perioperative care after thoracic and major abdominal surgery providing most effective analgesia.( ${ }^{(8)}$ It is possible to use single dose TEA for oncology mastectomies with axillary clearence, and this technique has many advantages when compared with general anesthesia.(4) TEA is associated with decreased incidence of nausea and vomiting and earlier discharge from the hospital compared to patients with general anesthesia. ${ }^{(9)}$ Successful use of high TEA avoids problems of difficult tracheal intubation, stress of anesthesia and surgery and hemodynamic changes associated with tracheal intubation. Stress associated with anesthesia and surgery results in increased catecholamine levels, increased left ventricular afterload, heart rate, and cardiac complications. TEA has been shown to decrease adverse perioperative cardiac events, morbidity and mortality after cardiac and non-cardiac major surgery. $(10,11)$

Autonomic neuropathy and macro vascular complications can lead to silent ischemia and myocardial infarction in long standing DM usually on the $2^{\text {nd }}$ or $3^{\text {rd }}$ post-operative day usually in the night. A segmental temporary sympathetic block provided by TEA is assumed to be an important beneficial factor of perioperative effects of TEA.(12,13) As patient was a known case of long standing type 2 DM and hypertension, we planned the procedure under sole anesthetic technique of TEA which provided safe and excellent surgical conditions. Patient tolerated the procedure well without any perioperative complications with additional benefit of prolonged postoperative analgesia. But TEA is also associated with complications hence one should always assess the risk and benefit ratio before planning the procedure. All the equipment and drugs should be kept ready to manage the complications and supplement general anesthesia if block fails or results in the inadequate block. Common complication of the technique is dural puncture, neurological injury and epidural hematoma9(14). With maximum precautions in experienced hand dural puncture is rare and incidence of neurological injury is $0.01-0.001 \% .(15)$

CONCLUSION: In conclusion, thoracic epidural anesthesia can be a better option to general anesthesia for modified radical mastectomy in high risk patients with long standing DM with co morbidities. Among the benefits of the technique includes a good postoperative analgesia, lower incidence of post-operative nausea and vomiting, shorter recovery time, and earlier hospital discharge. TEA is cost effective due to decrease in overall length of stay of the patient in the hospital.

\section{REFERENCES:}

1. Agarwal G, Ramakant P. Breast Cancer Care in India: The Current Scenario and the Challenges for the Future. Breast Care (Basel) 2003; 3 (1): 21-7.

2. Oddby-Muhrbeck E, Jakobsson J, Andersson L et al. -Postoperative nausea and vomiting. A comparison between intravenous and inhalation anaesthesia in breast surgery. Acta Anaesthesiol Scand, 1994; 38: 52-56. 


\section{CASE REPORT}

3. Stevenson GW, Hall SC, Rudnick S -The effect of anesthetiic agents on the human immune response. Anesthesiology, 1990; 72: 542-552.

4. Nesmith RL, Herring SH, Marks MW et al. -Early experience with high thoracic epidural anesthesia in outpatient submuscular breast augmentation. Ann Plast Surg, 1990; 24: 299-302

5. Ochroch EA, Gottschalk A, Augostides J et al. - Long term pain and activity during recovery from major thoracotomy using thoracic epidural analgesia. Anesthesiology, 2002; 97: 1234-1244.

6. Doss NW, Ipe J, Crimi T et al. Continuous thoracic epidural anesthesia with $0.2 \%$ ropivacaine versus general anesthesia for perioperative management of modified radical mastectomy. Anesth Analg, 2001; 92: 1552-1557.

7. Trikha A, Sadhasivam S, Saxena A, Arora MK, Deo SV. Thoracic epidural anesthesia for modified radical mastectomy in a patient with cryptogenic fibrosing alveolitis: a case report. J Clin Anesth 2000; 12 (1): 75-9.

8. Popping DM, Zahn PK, Van Aken HK, Dasch B, Boche R, Pogatzki-Zahn EM. Effectiveness and safety of postoperative pain management: a survey of 18925 consecutive patients between 1998 and 2006 ( $2^{\text {nd }}$ revision): a database analysis of prospectively raised data. Br J Anaesth 2008; 101:832-40.

9. Borgeat A, Ekatodramis G, Schenker C. -Postoperative nausea and vomiting in regional anesthesia; A review. Anesthesiology, 2003; 98; 530-547.

10. Beattie WS, Badner NH, Choi P. Epidural analgesia reduces post-operative myocardial infarction: a meta-analysis. Anesth Analg 2001; 93: 853-8.

11. Wijeysundera DN, Beattie WS, Austin PC, Hux JE, Laupacis A Epidural anesthesia and survival after intermediate to high risk non-cardiac surgery: apopulation based cohort study. Lancet 2008; 372: 562-9.

12. Meissner A, Rolf $\mathrm{N}$, Van Aken $\mathrm{H}$. Thoracic epidural anesthesia and the patient with heart disease: benefits, risks, and controversies. Anesth Analg 1997; 85: 517-28.

13. Clemente A, Carli F. The physiological effects of thoracic epidural anesthesia and analgesia on the cardiovascular, respiratory and gastrointestinal systems. Minerva Anestesiol 2008; 74: 54963.

14. Asegaonkar B N, Zine S R, Takalkar U V. Kulkarani U,. Asegaonkar S B, Kudlikeri P. Thoracic epidural anaesthesia for modified radical mastectomy in carcinoma of breast patient with chronic obstructive pulmonary disease: case report and Images (IJCRI) 4,10\{2013\};546-550.

15. Tanaka K, Watanabe R, Harada T, Dan K. Extensive application of epidural anesthesia and analgesia in a university hospital incidence of complication related to technique. Reg Anesth 1993; 18: 34-8. 


\section{CASE REPORT}

\section{AUTHORS:}

1. Vishwanath R. Hiremath

\section{PARTICULARS OF CONTRIBUTORS:}

1. Professor, Department of Anesthesiology and Critical Care, Sri Lakshmi Narayana Institute of Medical Sciences, PuducherryAffiliated to Bharath University, Chennai.
NAME ADDRESS EMAIL ID OF THE CORRESPONDING AUTHOR:

Dr. Vishwanath R. Hiremath,

Department of Anesthesiology and

Critical Care,

Sri Lakshmi Narayana Institute of Medical Sciences, Puducherry-605502,

Affiliated to Bharath University, Chennai.

Email:vishwanath0506@gmail.com

Date of Submission: 01/12/2014.

Date of Peer Review: 02/12/2014.

Date of Acceptance: 08/12/2014.

Date of Publishing: 15/12/2014. 